\title{
Poly(vinyl methyl ether)/Poly(methyl methacrylate) Blends Using Diblock Copolymer of Styrene and Methyl Methacrylate as Compatibilizer
}

\author{
Hanying ZhaO, ${ }^{\dagger}$ Ming GaO, and Baotong Huang \\ Changchun Institute of Applied Chemistry, Chinese Academy of Sciences, \\ Changchun, 130022, People's Republic of China
}

(Received December 3, 1996)

\begin{abstract}
Blends of poly(vinyl methyl ether) (PVME) and poly(methyl methacrylate) (PMMA) compatibilized by poly(styrene-block-methyl methacrylate) (P(S-b-MMA)) are studied by FT-IR, DSC, excimer fluorescence spectrometry, and scanning electron microscopy (SEM). In FT-IR measurement the ratio of absorption intensity at $1107 \mathrm{~cm}^{-1}$ to that at $1085 \mathrm{~cm}^{-1}$ $\left(I_{1107} / I_{1085}\right)$ reaches a minimum at about $10 \mathrm{wt} \%$ block copolymer content. DSC results show that the glass transition temperature of PVME in the blends has a maximum at $10 \mathrm{wt} \%$ copolymer content. In plots of the ratio of excimer-to-monomer fluorescence emission intensities $\left(I_{\mathrm{E}} / I_{\mathrm{M}}\right) v s$ block copolymer content, $I_{\mathrm{E}} / I_{\mathrm{M}}$ increases rapidly above $10 \%$. All these phenomena show that PS block chains penetrate into PVME domains on addition of block copolymer. Above $10 \%$ copolymer content, block copolymer chains tend to form micelles in bulk phase. KEY WORDS Poly(vinyl methyl ether) / Poly(methyl methacrylate) / Poly(styrene-block-methyl methacrylate) / Compatibilizer / Interface / Micelles /
\end{abstract}

Blends of two or more polymers create new materials with desirable combination of properties. Unfortunately, in most cases blending is very difficult or impossible because of thermodynamical immiscibility. Blends of immiscible polymers will give poor interfacial adhesion and poor mechanical properties. Addition of block or graft copolymers to immiscible polymers blends can modify the state of the interface, reducing interfacial tension and improving interfacial adhesion. ${ }^{1-3}$

Block or graft copolymers, which are chemically identical to or miscible with homopolymers at each block or graft, are distributed at the interface and reduce the interfacial energy between the phases, providing a finer dispersion of the discontinuous phase. Block or graft copolymer chains in immiscible homopolymer blends also favor micellar aggregation in homopolymer matrix besides their location at the interface. ${ }^{4,5}$ The micelles compete with the interfacial region for copolymer chains, and the amount in each state depends on the relative reduction in free energy as well as the interface area. The formation of micelles may lower the efficiency of compatibilization.

The use of block copolymers as compatibilizers often does not result in an improvement of the mechanical properties, though the interfacial tension is reduced. The mechanical properties of a blend depend not only on the reduction of the interfacial tension but also on the extensive overlap and entanglement of the copolymers with the appropriate homopolymer domains. ${ }^{6}$ If the block copolymer and homopolymer chains do not interpenetrate (dry brush), the interfacial adhesion will be very weak. Only when the compatibilizer mixes with the blend components at the interface (wet brush), an efficient mechanical coupling will be achieved. The molecular weight of the blend components and ratio of block lengths influence the balance of the dry brush and wet brush. ${ }^{7,8}$ The negative enthalpic interaction between a block copolymer and homopolymer favors in-

\footnotetext{
${ }^{\dagger}$ To whom correspondence should be addressed.
}

terpenetration and the wet brush situation.

In toluene-cast blends, polystyrene (PS) and poly(vinyl methyl ether) (PVME) are miscible. ${ }^{9-13}$ This is due to interaction between the ether lone pairs of electrons and the benzene rings of PS. ${ }^{11}$ So poly(styrene-block-methyl methacrylate) (P(S- $b$-MMA)) should serve well as a compatibilizer for blends of PVME/poly(methyl methacrylate) (PMMA).

\section{EXPERIMENTAL}

\section{Materials}

$\mathrm{P}(\mathrm{S}-b$-MMA) was prepared by sequential anionic polymerization in tetrahydrofuran (THF) at $-78^{\circ} \mathrm{C}$. The basic equipment consisted of a $400 \mathrm{ml}$ stirred, argon pressurized glass reactor. THF was predried by refluxing over $\mathrm{CaH}_{2}$ and $\mathrm{KOH}$ for at least 10 hours, further purified by refluxing over potassium and sodium for 1 week and then distilled directly into an ampule. MMA was purified by standard procedures ${ }^{14}$ with $\mathrm{Et}_{3} \mathrm{Al}$. Styrene was distilled over $\mathrm{CaH}_{2}$ at least 3 times.

Polymerization of styrene was initiated with $n$-BuLi. After the desired amount of styrene was slowly added, the PS block was capped with 1,1-diphenylethylene and then MMA was added dropwise at $-78^{\circ} \mathrm{C}$. The polymerization was terminated by alcohol. Finally the copolymer was precipitated by pouring the mixture into 5-fold excess of alcohol.

The molecular weight of $\mathrm{P}(\mathrm{S}-b$-MMA $)$ was $5.928 \times 10^{4}$ $\left(M_{w}\right)$; the polydispersity was 1.25 (measured by GPC on PS standards). The content of MMA was $40 \mathrm{~mol} \%$ measured by ${ }^{1} \mathrm{H}$ NMR.

PMMA was also synthesized by anionic polymerization in THF at $-78^{\circ} \mathrm{C}$. The polymerization of MMA proceeded by adding 1,1-diphenylethylene to $n$-BuLi at $-78^{\circ} \mathrm{C}$. The polymerization was also terminated by alcohol and the solution was poured into alcohol. The molecular weight of the isolated PMMA was $1.823 \times 10^{4}$ $\left(M_{w}\right)$, the polydispersity being 1.21 .

Poly(vinyl methyl ether) with a molecular weight of 
$6.3 \times 10^{4}\left(M_{w}\right)$ and polydispersity 1.7 was purchased from Polyscience.

\section{Blend Preparation}

Blends were prepared by dissolving the components (the weight ratio of PVME/PMMA is $1: 2.33$ ) in toluene $(\sim 3 \mathrm{wt} \%)$, evaporating the solvent slowly in a Teflon cell, drying the samples in vacuo for a week at $90^{\circ} \mathrm{C}$.

Fourier Transform Infrared Spectroscopy Measurement

The samples for FT-IR measurement were prepared by coating polymer solution on $\mathrm{KBr}$ plates, evaporating the solvent slowly and drying the sample in vacuo for a week at $90^{\circ} \mathrm{C}$. Infrared studies were done on a BIO-RAD FTS-7 Fourier transform infrared (FT-IR) spectrometer at a resolution of $1 \mathrm{~cm}^{-1}$.

\section{Fluorescence Measurements}

The samples used in the fluorescence study were prepared by coating the solution on squartz plates, evaporating the solvent and drying in vacuo for a week at $90^{\circ} \mathrm{C}$. Emission spectra were measured on a Shimadzu RF-5000 fluorescence spectrometer at room temperature. Peak heights of the fluorescence intensities at $\lambda=$ 330 and $\lambda=285 \mathrm{~nm}$ with $\lambda=260 \mathrm{~nm}$ excitation were taken as $I_{\mathrm{E}}$ and $I_{\mathrm{M}}$, respectively.

\section{Differential Scanning Calorimetry Measurements}

Differential scanning calorimetry (DSC) measurements were performed using a Perkin-Elmer DSC-2C thermal analyzer at a heating rate of $20^{\circ} \mathrm{C} \mathrm{min}^{-1}$ under nitrogen atmosphere. The temperature range was -50 $150^{\circ} \mathrm{C}$.

\section{SEM Observation}

The fracture surface for SEM observation was prepared by fracturing the sample frozen in liquid nitrogen for 5 mins. The morphology of the fractured surface was observed on a JEOL-MAX-840 scanning electronic microscope after coating with gold.

\section{RESULTS AND DISCUSSION}

\section{Fourier Transform Infrared Spectroscopy}

FT-IR spectroscopy is a powerful tool for studying polymer blends on molecular level. ${ }^{15}$ The position, intensity, and shape of peaks are useful in identifying conformational and environmental changes of polymers. A distinct chemical interaction between two polymers in a blend will result in difference in spectra of a polymer in the blend and that of pure components. Lu et al. ${ }^{10}$ studied the PS/PVME blends by FT-IR spectroscopy and found that PVME had a strong doublet at $1085 \mathrm{~cm}^{-1}$ with a shoulder at $1132 \mathrm{~cm}^{-1}$ and the $1085 \mathrm{~cm}^{-1}$ peak was stronger in miscible blends whereas the $1107 \mathrm{~cm}^{-1}$ peak stronger in immiscible blends.

Our FT-IR results show that in the immiscible PVME/ PMMA blends, the $1107 \mathrm{~cm}^{-1}$ peak predominates (Figure 1). However, the difference between the intensities of $1107 \mathrm{~cm}^{-1}$ and $1085 \mathrm{~cm}^{-1}$ gets smaller on addition of P(S- $b$-MMA). $I_{1107} / I_{1085}$ reaches a minimum at about $10 \%$ block copolymer content. When the concentration of block copolymer is above $10 \%, I_{1107} / I_{1085}$ increases

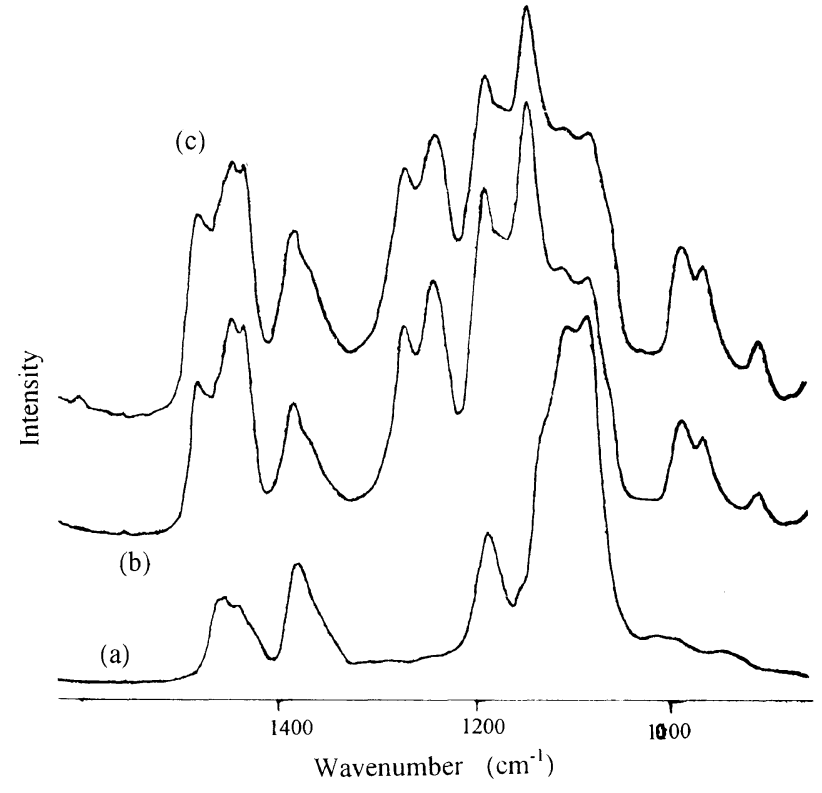

Figure 1. Fourier transform infrared spectra of PVME (a) and blends of PVME and PMMA $(1: 2.33)$ with varying amounts of diblock copolymer. (b), containing $0 \mathrm{wt} \%$ diblock copolymer; (c), containing $2.7 \mathrm{wt} \%$ block copolymer.

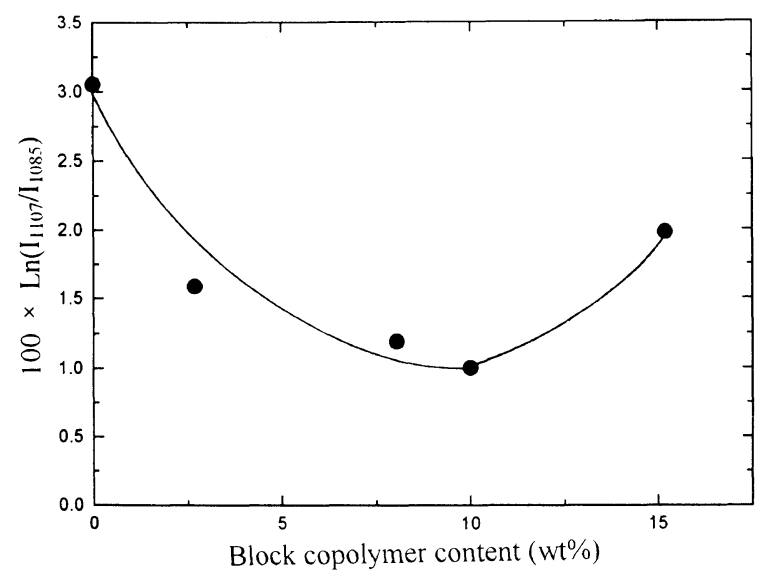

Figure 2. Relationship of $I_{1107} / I_{1085}$ with the weight percent of diblock copolymer.

again (Figure 2).

In compatibilized blends, at low block copolymer concentration diblock copolymer chains migrate to the interface forming a "brush."16 With increase in copolymer concentration, more block copolymer chains segregate at the interface, and the interfacial tension is reduced. Because of the exothermic and interaction between PS and PVME, PS block is expected to penetrate into PVME domains. Change of relative intensity at 1085 and $1107 \mathrm{~cm}^{-1}$ reflects essentially this change of PVME molecular environment near the interface. At higher copolymer concentration (above $10 \mathrm{wt} \%$ ), block copolymer chains tend to form micelles in the bulk phase.

In which of the bulk phases is micelle formation more likely to occur? If most micelles exist in PVME phase, PS chains, as corona of micelles, would penetrate into PVME chains, and because of this change of PVME molecular environment the value of $I_{1107} / I_{1085}$ would not increase at high copolymer content. So we can 
ascertain that most micelles exist in PMMA phase, in which PS chains of block copolymer act as core and PMMA chains as corona. Only in this way PS chains would not interact with PVME chains.

\section{$T_{\mathrm{g}}$ Behavior}

The glass transition temperature of PVME is far below that of PMMA and PS, so DSC can be used to examine the diffusion of PVME chains in the interface. The glass transition behavior of blends of PVME/PMMA compatibilized by $\mathrm{P}(\mathrm{S}-b$-MMA) as a function of block copolymer content is shown in Figure 3. On addition of the block copolymer the glass transition temperature of PVME increases. It is apparent that PVME chains diffuse to the interface and are miscible with PS chains in the interface. According to Bank's report, ${ }^{17}$ for binary blends PS/PVME the experimental data have a best fit with the Gordon-Taylor expression

$$
T=\frac{k C_{2} T_{2}+C_{1} T_{1}}{C_{1}+k C_{2}}
$$

where $C_{1}$ and $C_{2}$ are weight fraction of polymer 1 and polymer 2 , respectively, $T_{1}$ is $T_{\mathrm{g}}$ of polymer $1, T_{2}$ is $T_{\mathrm{g}}$ of polymer $2, T$ is $T_{\mathrm{g}}$ of the mixture, and $k$ is a constant (0.2).

Referring to the Gordon-Taylor expression, the observed points are close to the predicted transition temperatures at low copolymer contents. But large deviations occur at higher copolymer contents, especially at $15 \mathrm{wt} \%$ block content. The glass transition temperature of PVME has a maximum at $10 \mathrm{wt} \%$ copolymer content. At low copolymer content, most block copolymer chains distribute at the interface and PS chains of the block copolymer interpenetrate into PVME domains. So experimental data fit well with predicted results. Above $10 \mathrm{wt} \%$ copolymer content, most copolymer chains form micelles. It is noted that $T_{\mathrm{g}}$ decreases above $10 \%$ copolymer content, which suggests that most micelles exist in PMMA phase but not PVME phase. If most micelles exist in PVME phase, glass transition temperature of PVME would keep increasing above $10 \%$ because of the miscibility of PVME chains with PS chains at corona of micelles.

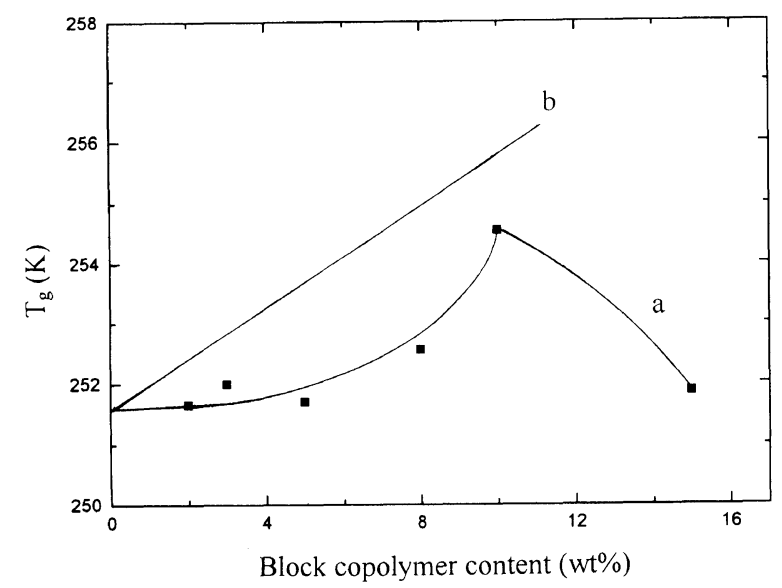

Figure 3. Glass transition temperature of PVME by DSC at $20^{\circ} \mathrm{C} \mathrm{min}^{-1}$ versus concentration of block copolymer. Curve a, experimental data; curve $b$, results calculated according to GordonTaylor expression.
It is observed that in the ternary blends glass transition gets broadened upon addition of the block copolymer (Figure 4). This indicates that the composition varies across the interface. The PS segment density decreases from a high value near the interface to a low value in the inner regions of the PVME phase, i.e., the interface gets broadened.

\section{Excimer Fluorescence Behavior}

As excimer interaction functions in a very short range, as small as several nanometers, it is expected to provide structural information of small sized domains. Frank et $a l .^{18-21}$ and Qian et $a .^{22}$ studied PS/PVME blends using excimer fluorescence and found PS and PVME are miscible in segmental level.

Our experimental results (Figure 5) show $I_{\mathrm{E}} / I_{\mathrm{M}}$ increases linearly up to $10 \%$ copolymer weight fraction and at high copolymer content $I_{\mathrm{E}} / I_{\mathrm{M}}$ increases rapidly. It is very interesting to compare our results with those by Qian ${ }^{22}$ and Frank ${ }^{23}$ for binary blends. Their work showed that for toluene-cast binary miscible PS/PVME blends $I_{\mathrm{E}} / I_{\mathrm{M}}$ was constant at low PS concentrations $(<5 \mathrm{wt} \%)$, which means that in compatible PS/PVME blends the chain segments of PS and PVME are dispersed in segmental level and energy migration is one dimensional, energy transfer occurring mainly between adjacent chromophores. At high PS concentration, excimers can form between chromophores on different chains, or energy transfer between chromophores on different chains becomes possible. In ternary PVME/ PMMA/P(S- $b$-MMA) blends, the linear relationship of

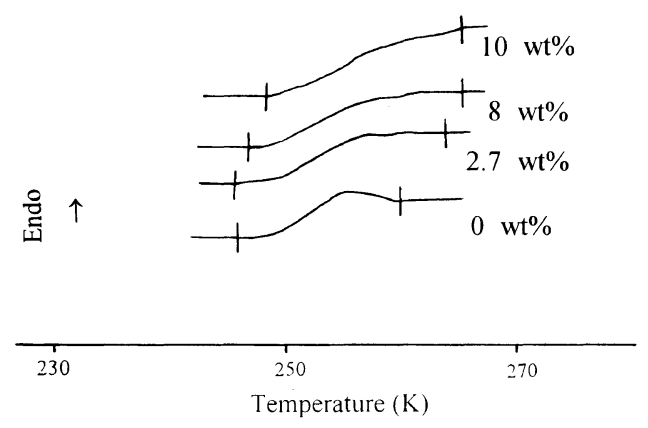

Figure 4. Representative DSC thermograms for the transition region of PVME in blends with PMMA at different block copolymer concentration.

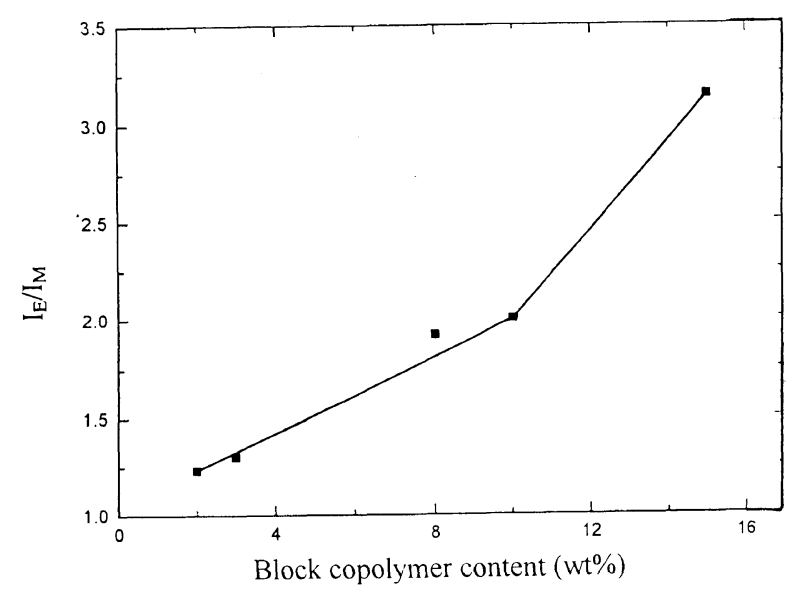

Figure 5. Change of $I_{\mathrm{E}} / I_{\mathrm{M}}$ with addition of block copolymer. 

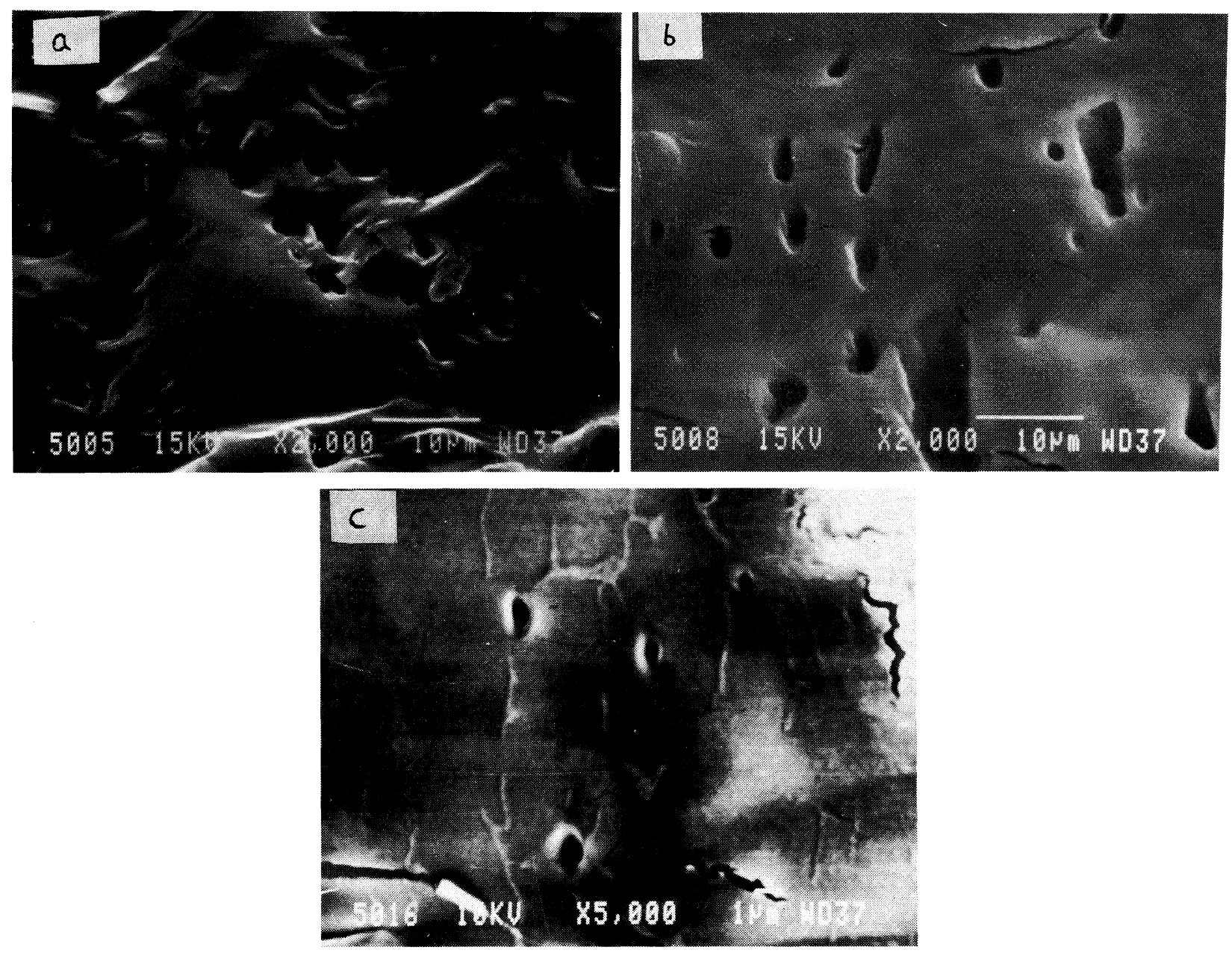

Figure 6. SEM micrographs of morphology of fracture surface of PVME/PMMA $=1: 2.33$ (W/W) blends compatibilized by $\mathrm{P}(\mathrm{S}-b$-MMA). (a) blends without block copolymer; (b) blends with $6 \mathrm{wt} \%$ block copolymer; (c) blends with 10 wt $\%$ block copolymer.

$I_{\mathrm{E}} / I_{\mathrm{M}} v s$. block copolymer concentration suggests that PS and PVME in the interface are interpenetrated in segmental level and the PS segment density at the interface increases with addition of the block copolymer. At higher block copolymer content, most block copolymer chains tend to form micelles in PMMA phase, the aggregation of PS chains in the core leads to energy transfer between chromophores on different chains. So excimer fluorescence measurement serves as direct evidence for the formation of micelles in PMMA phase at high block copolymer contents.

\section{SEM Observation}

Scaning electron microscopy is frequently used in the study of polymer blends with compatibilizers. In our study SEM observation was also carried out. The fracture surface morphology of PVME/PMMA blends with 0,6 , and $10 \mathrm{wt} \%$ diblock copolymer was studied by SEM (Figure 6). We can see that the size of dispersed particles get smaller with addition of copolymer, which indicates the decrease of interfacial tension between PVME phase and PMMA phase.

Dai et al. ${ }^{16}$ used forward recoil spectrometry (FRES) to study the effects of molecular weight of homopolymer on interfacial segregation in two-phase blends containing diblock copolymers. They found that if the degree of polymerization of homopolymer $\mathrm{A}$ is higher than

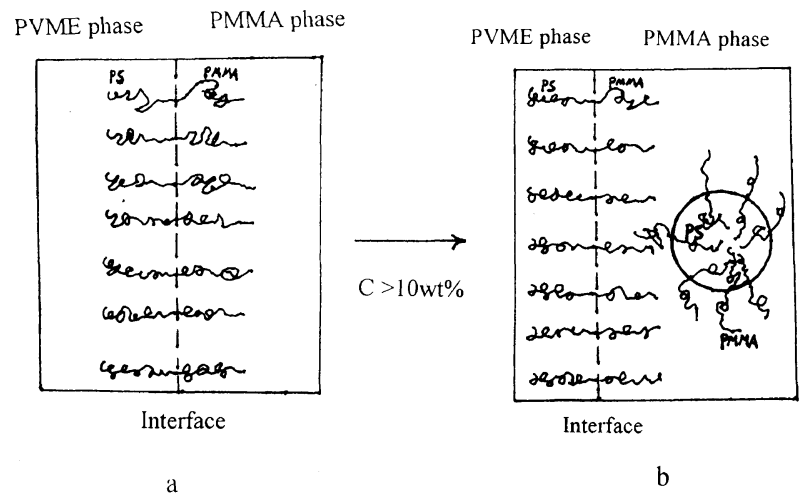

Figure 7. Schematic illustration of PVME/PMMA blends compatibilized by $\mathrm{P}(\mathrm{S}-b$-MMA). a: Block copolymer chains distribute at the interface at low copolymer content. $b$ : Block copolymer chains form micelles when block copolymer concentration is above $10 \mathrm{wt} \%$.

that of the A block of the copolymer, the interfacial areal density of block copolymer chains at saturation is not affected by the homopolymer. This means that the "brush" of A block chains is not penetrated by homopolymer A (the dry brush case). If the molecular weight of the homopolymer is smaller, homopolymer chains migrate to the interface and penetrate the "brush" of A block chains.

In our experiment, the molecular weight of homo- 
PMMA is smaller than that of the PMMA block in the block copolymer. So homopolymer chains penetrate the PMMA "brush" at the interface or a wet brush is created.

A physics model of compatibilization for this system is shown in Figure 7. At low block copolymer content, block copolymer chains are distributed at the interface. PS block chains interpenetrate into PVME phase and PMMA block chains interpenetrate into PMMA phase. When block copolymer content is above $10 \mathrm{wt} \%$, block copolymer chains form micelles in PMMA phase. PS block chains exist in the core and PMMA block chains act as corona of the micelles. Because the molecular weight of homo-PMMA is smaller than that of the PMMA block, homo-PMMA chains can migrate to the shell of the micelles and interact with PMMA block chains, or the corona of micelles can be swollen by homopolymer chains. ${ }^{24}$

\section{CONCLUSION}

The results of FT-IR, DSC, excimer fluorescence and SEM show that because of the miscibility of PVME with PS, the PS block has a thermodynamic driving force to diffuse into the PVME phase. As the block copolymer content increases, the interface broadenes and PS segment density at the interface increases. At high copolymer concentration, block copolymer chains tend to form micelles in PMMA phase. Because the molecular weight of homo-PMMA is lower than that of the PMMA block, homo-PMMA chains are also able to diffuse into the interface.

Acknowledgment. This project was supported by National Nature Sciences Foundation of China and Polymer
Physics Laboratory in Changchun Institute of Applied Chemistry, Chinese Academy of Sciences.

\section{REFERENCES}

1. D. R. Paul and S. Newman, "Polymer Blends," Vol. 2, Academic Press, New York, N.Y., 1979, p. 35.

2. J. Noolandi, Polym. Eng. Sci., 24, 70 (1984)

3. J. M. Huet and E. Marechal, Eur. Polym. J., 10, 771 (1974).

4. T. Inoue, T. Soen, T. Hashimoto, and H. Kamai, J. Polym. Sci., Part $A-2,7,1283$ (1969).

5. J. Noolandi and K. M. Hong, Macromolecules, 15, 482 (1982).

6. H. H. Kausch and M. Tirrel, Annu. Rev. Mater. Sci., 19, 341 (1989).

7. L. Leibler, Makromol. Chem., Makromol. Symp., 16, 1 (1988).

8. J. Noolandi and K. M. Hong, Macromolecules, 17, 1531 (1984).

9. J. Noolandi and K. M. Hong, Macromolecules, 4, 44 (1971).

10. F. J. Lu, E. Benedetti, and S. L. Hsu, Macromolecules, 16, 1525 (1983).

11. D. Garcia, J. Polym. Sci., Polym. Phys. Ed., 22, 1773 (1984).

12. E. Jabbari and N. A. Peppas, Polym. Internat., 38, 65 (1995).

13. E. Jabbari and N. A. Peppas, Macromolecules, 28, 6229 (1995).

14. R. D. Allen, T. E. Long, and J. E. Mcgrath, Polym. Bull., 15, 127 (1986).

15. M. M. Coleman and P. C. Painter, Appl. Spectrosc. Rev., 20, 255 (1984).

16. K. H. Dai, E. J. Kramer, and K. R. Shull, Macromolecules, 25, 220 (1992).

17. M. Bank, J. Leffingwell, and C. Thies, Macromolecules, 4, 42 (1971).

18. S. N. Semerak and C. W. Frank, Macromolecules, 14, 443 (1981)

19. M. A. Gashgari and C. W. Frank, Macromolecules, 14, 1558 (1981).

20. P. D. Fitzgibbon and C. W. Frank, Macromolecules, 15, 733 (1982).

21. R. Gelles and C. W. Frank, Macromolecules, 15, 1486 (1982).

22. R. Qian and X. Gu, Angew. Makromol. Chem., 141, 1 (1986).

3. R. Gelles and C. W. Frank, Macromolecules, 15, 747 (1982).

24. M. D. Whitmore and J. Noolandi, Macromolecules, 18, 657 (1985). 